\title{
TUP1-mediated filamentation in Candida albicans leads to inability to colonize the mouse gut
}

Elvira Román, Blanca Huertas, Daniel Prieto, Rosalía Díez-Orejas and Jesús Pla*

Departamento de Microbiología y Parasitología, Facultad de Farmacia, Universidad Complutense de Madrid, Plaza de Ramón y Cajal s/n, E-28040 MADRID, SPAIN

Phone: + 34913941617

FAX: + 34913941745

*Corresponding author: jesuspla@farm.ucm.es

Keywords

Candida albicans / filamentous growth / commensalism / TUP1

Abbreviations

SD, standard deviation, aCT. Autoclaved chlortetracycline. 


\section{Abstract}

Aims: To investigate the role of Candida albicans TUP1-mediated filamentation in the colonization of the mice gut. Material \& Methods: We use molecular genetics to generate a strain where filamentation is regulated by altering the expression of the TUP1 gene with tetracyclines. Results: The colonization rates reached with the TUP1REP-RFPREP strain were lower compared to wild type strain and completely absent after induction of filamentation. No differences in the susceptibility to bile salts nor in the adhesion to the mouse intestine epithelium was observed. Conclusions: Locking the blockage of $C$. albicans in a filamentous form impedes cells gut colonization in the mouse. 


\section{Introduction}

Candida albicans is a pathogenic yeast that causes a wide range of infections, collectively called candidiasis. This yeast is part of the microbiota of most humans but depending on the immunological status of the host it may lead to systemic diseases on immune deficient individuals that are very severe and frequently life-threatening. This yeast is therefore considered an opportunistic pathogen, residing in the oral cavity and the gastrointestinal \& urogenital tracts of immunocompetent individuals. C. albicans pathogenesis is considered to be multifactorial, as there is not a single factor responsible for the virulence of this fungus; rather, several components have been shown to be involved in its pathogenicity such as metabolic traits, signalling pathways, cell wall architecture and secreted products that make separate local and temporal contributions during pathogenesis [1,2]. The relevance of most of these factors has been determined using either in vitro or in vivo models. Among the later, the intravenous systemic infection model has been the most widely used in the past for testing virulence determinants in this yeast [3] although superficial and mucosal models are clearly relevant considering the pathology of candidiasis [4]. The use of commensalism models developed long time ago [5,6], but only popularized recently [7,8], is enabling the analysis of the contribution of different genes to colonization [9].

Several $C$. albicans factors have been shown to play a role in the adaption to the commensal state during gastrointestinal colonization $[9,10]$. They comprise epigenetic transitions [11], iron acquisition [12], signalling pathways [13] and metabolic traits [14] among others. One important feature of $C$. albicans is its dimorphic nature, the ability (under appropriate stimuli) to develop a yeast to hyphae transition. This feature has been related to its ability to cause infections: $C$. albicans mutants strains that grow only in the yeast form were found to be avirulent in the mouse systemic model [15], although mutant strains that only grew -or had an enhanced ability to grow- in the filamentous forms were also found to be attenuated in virulence $[16,17]$. It is now generally accepted that neither of these forms are intrinsically virulent although they may play different specialized functions during infection within the host, both temporally and locally.

Although dimorphism has been mainly studied in the mouse systemic model [18], its role in the mouse commensalism model is less documented. An early study made use of an intrinsically filamentous mutant (tup1) that revealed reduced colonization in the gut [7]. Also, a recent study using strains locked in the filamentous form a similar behaviour [19]. However, both studies suffered from using intrinsically filamentous forms of $C$. albicans, which are difficult to handle in the laboratory due to its hyphal nature which does not easily allow standardization of inoculum administration. In addition, as the inoculum is administered as a complete hyphal form, and one could argue that hyphi are not deficient in long term colonization but only during the early stages that mediate adaptation to the gut cavity [20]. To circumvent these caveats and allow a temporal dissection of this process, we have generated a $C$. albicans mutant strain in which filamentation can be triggered by altering TUP1 levels, as deletion of this transcriptional regulator lock cells in the filamentous form [21]. Using a filamentation doxycycline regulated strain we have determined that induction of filamentation once established in the mouse gut results in the inability to colonize the mouse gut and complete loss of the fungus. We have also validated the use of a heat inactivated chlortetracycline using functional bioassays, showing its usefulness in in vivo analysis of gene expression in this fungus.

\section{Strains and growth conditions}

The strains used are described in Table 1. Cells were grown at $37^{\circ} \mathrm{C}$ in YPD medium ( $1 \%$ yeast extract, $2 \%$ peptone and $2 \%$ dextrose) unless otherwise stated. Chlortetracycline (Sigma-Aldrich) was heat inactivated 


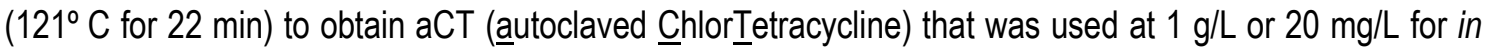
vivo and in vitro assays respectively.

The susceptibility/resistance to different compounds was performed through drop test as follows. Cultures grown at $37^{\circ} \mathrm{C}$ for 16 hours, in the presence or not of aCT, were adjusted to $2 \times 10^{7}$ cells $/ \mathrm{mL}$, serially 10 -fold diluted and deposited $(5 \mu \mathrm{l})$ onto solid YPD plates supplemented (or not) with bile salts (Sigma-Aldrich). Plates were incubated at $37^{\circ} \mathrm{C}$ for 24 hours before scanned.

\section{Genetic procedures}

To develop a regulated filamentous strain, the ORF of TUP1 was amplified by PCR from the clinical isolate SC5314 strain using the primers up-TUP1-myc (GATCTCGAGTATGTATCCCCAACGCACCC) and revTUP1-myc (ATCGCGGCCGCATTTTTTTGGTCCATTTCCAAATTC). The 1536 bp PCR product was cloned in the intermediate pGEMT plasmid (Promega), digested with Xho I and Not $I$ and accommodated in pNRURFP [22], a doxycycline repressible plasmid, previously digested with Sal I and Not I to generate pNRUTUP1, were TUP1 is tagged with the myc epitope. Homologous recombination occurs at the ADH1 locus by means of Kpn I-Sac II digestion. Transformation of C. albicans in a tup1 background [21] generated a TUP1REP strain. For RFP labeling, we used the pDS2-dTOM2 plasmid, which expresses, together with pNRU, express RFP under the control of the TET promoter and recombines at the ARD1 locus by Kpn I digestion. It was constructed as follows: the TET promoter (tetO+OP4) was amplified by PCR from pNIM1 plasmid using the primers TETPR up (CTAGTCGACTTTACCACTCCCTATCAGTGATAGAGAA) and TETPR rev (GTAGCTAGCTATTTATATTTGTATGTGTGTAGGAGTTAACG), digested with Sal I and Nhe I and introduced in the plasmid pDUMO-L [23], previously digested with Xho I and Nhe I, to generate the pDUM2$L$ plasmid. The dTOM2 ORF was amplified with the primers dTOM2 up (ACTGCTAGCGAGATGGTTTCTAAAGGTGAAGAATCG) and dTOM2 rev (AAGGATCCTCGAGCCCATATTATTATCTTCAGAAGAAGCAGTACC) from pNIM1R-dTOM2 [13], digested with Nhe I and BamH I and introduced in PDUM2-L by replacing the CbLUC gene to generate pDUM2-dTOM2. Finally, the SAT1 marker was amplified from pNIM1 with the primers SAT1_up (ACTTCTAGATGTCTGCAGGACCACCTTTGATTG) and SAT1_rev (CTGCCGCGGAGCGTCAAAACTAGAGAATAATAAAGAAAACG), digested with Xba I and Sac II and ligated in pDUM2-dTOM2, digested with the same pair of enzymes, replacing the URA3 marker, to generate the final pDS2-dTOM2 plasmid.

\section{Protein extracts and immunoblot analysis}

All procedures involving cell lysis, protein extraction, gel electrophoresis and transfer to nitrocellulose membranes were made as previously described [24]. Protein extracts were measured at $A_{280 \mathrm{~nm}}$ to equalize the amount of protein loaded for western blot analysis and probed with anti-myc, clone 4A6 (Millipore). Western blots were developed according to the manufacturer's conditions using the Hybond ECL kit (Amersham Pharmacia Biotech).

\section{In vivo fitness assays}

The murine gut colonization assays were performed following the protocol previously described [13]. Briefly, after 4 days of antibiotic pre-treatment $(2 \mathrm{mg} / \mathrm{mL}$ streptomycin, $1 \mathrm{mg} / \mathrm{mL}$ bacitracin, and $0.1 \mathrm{mg} / \mathrm{mL}$ gentamycin) with or without $\mathrm{aCT}, 10^{7} \mathrm{C}$. albicans cells were intragastrically inoculated in a single gavage dose. Stool samples were obtained on different days (indicated in the accompanying figures) and homogenized in PBS prior to be cultured in SD plates to determine CFUs per gram. 4 mice were used in 
each experiment and repeated at least twice; only a representative experiment is shown. To analyze C. albicans loads in the gastrointestinal tract, mice were sacrificed and intestinal content from stomach, cecum, small and large intestine were aseptically obtained, homogenized in sterile PBS and cultured in SD plates to count CFUs. Female mice C57BL/6 were purchased from Harlan Laboratories, Inc. (Italy) and used within an age of 7 to 10 weeks-old. Mice housing and other non-invasive procedures took place in the animal housing facility from the Medical School of the Universidad Complutense de Madrid.

All experiments involving animals performed in this work were carried out in strict accordance with the regulations in the "Real Decreto 1201/2005, BOE 252" for the Care and Use of Laboratory Animals of the "Ministerio de la Presidencia," Spain. The protocol used in the commensalism model was approved by the Animal Experimentation Committee of the University Complutense of Madrid (CEA 33-2015) and Comunidad de Madrid according to Artículo 34 del RD 53/2013 (PROEX 226/15). The treatments did not result in noticeable disease in the animals; nevertheless, all procedures were conducted minimizing any suffering. The number of animals per experiment was adjusted to a minimum for ethical reasons. Experiments were done at least twice and only one representative experiment is shown in the Figures.

\section{Adhesion assays}

Adhesion to the intestinal mucosa was assessed as previously described [13]. Briefly, a $1 \mathrm{~cm}$-piece of the large intestine of a non-colonized euthanized mouse was opened, washed gently with PBS and placed in a $4 \mathrm{~mm}$-diameter methacrylate chamber made of two different sheets of methacrylate joined by clamps. The chamber was immediately filled with RPMI medium pre-warmed at $37^{\circ} \mathrm{C}$. Then, two $\mathrm{C}$. albicans strains (from overnight YPD cultures) to be tested were mixed (1:1 ratio) and adjusted to $2 \times 10^{6} \mathrm{cells} / \mathrm{mL}$ concentration in serum-free RPMI medium. $10^{5}$ yeast cells from this suspension were placed facing the original lumen of the colonic tissue in the chamber and incubated for $150 \mathrm{~min}$ at $37^{\circ} \mathrm{C}$. After this period, the piece of tissue was carefully washed twice with PBS and mechanically disaggregated. Serial dilutions of this last fraction was spread on SD media for CFUs determination. Three similar (size, region) pieces of colonic tissue were used for each competitive determination.

An internal control (CAF2-GFP [13]) was introduced in all adhesion assays; therefore, adherence is quantified by the adhesion relative index (ARI). This index is calculated by dividing the relative amounts of the strain under analysis in adhered cells relative to the value of that same strain in the inoculum and therefore reflects the adhesion relative to the parental CAF2-GFP strain.

\section{Statistical analysis}

Statistical differences were assessed with the SPSS Software (UCM, Madrid, Spain). A $p<0.05$ was considered significant using ANOVA tests (followed by Dunett's multiple comparisons test).

\section{Results}

\section{Development of a filamentation - regulatable strain by tetracyclines}

In order to analyse the role of the yeast-to-hypha transition, we constructed a strain in which the TUP1 gene is under the control of the tetracycline repressible version (TET-OFF). For this purpose, the TUP1 coding region was cloned in the pNRU plasmid (see Material and Methods), which also tagged the protein with a 
c-terminal myc epitope, and the gene reporter RFP (dTOM2) in the pDS2 plasmid. The constructs were integrated in a tup1 mutant generating the TUP1REP-RFPREP strain. To confirm the conditional expression system, we analysed TUP1 function by observation of the expected filamentous characteristic phenotype of tup1 mutants in the TUP1REP -RFPREP strain both in liquid and solid media. Under normal conditions (YPD without antibiotic), only yeast cells were observed (Figure 1A). However, the addition of doxycycline to the medium induced the filamentation due to the repression of TUP1 and wrinkled colonies appeared on solid agar plates. In both cases, the wild type CAF2 strain showed the corresponding yeast phenotype while a homozygous tup1 mutant remained permanently blocked in the filament form.

Due to the broad antibacterial spectrum of doxycycline and its potential effect on mice microbiota during in vivo experiments, which could interfere with the results, we tested a heat inactivated variant of tetracycline (autoclaved chlortetracycline, aCT) [13, 25]. To validate the use of aCT in colonization experiments, we determined in vitro its antimicrobial activity (as determined by CMI measures) against $E$. coli DH5a strain compared to the non-inactivated doxycycline. aCT resulted in a 30x fold reduction in its CMI compared to non-inactivated doxycycline (1.5 mg/L vs $50 \mathrm{mg} / \mathrm{L}$ ) (data not shown). On the other hand, aCT efficiently repressed TUP1 expression in TUP1REP-RFPREP strain similarly to doxycycline, since both compounds showed the same efficiency in inducing filamentation (via TUP1 repression) when concentration reaches $0.05 \mathrm{mg} / \mathrm{L}$ where hyphae and pseudohyphae were detected (Figure 1B).

We also analysed if this effect was due to the repression of TUP1. We studied the ability of aCT and doxycycline to repress TUP1 by western blot (Figure 1C). In the absence of antibiotic, a clear band of Tup1myc with the expected molecular weight of $59 \mathrm{kDa}$ appeared while no band was observed when antibiotic ( $>0.05 \mathrm{mg} / \mathrm{L}$ ) was added to the medium indicating the tight regulation of both compounds.

\section{Role of TUP1-mediated filamentation in mice gut colonization}

The ability of aCT to regulate cellular morphology via TUP1 enabled us to determine the contribution of the filamentous form in the colonization of mice gut. To validate $\mathrm{aCT}$ as a tool to regulate gene expression in vivo without alteration in the gut fitness, we analyse $\mathrm{aCT}$ bioavailability and activity along mouse intestine. aCT $1 \mathrm{~g} / \mathrm{L}$ was incorporated in the drinking water of mice for 7 days; the animals were then sacrificed and intestinal samples were used in a bioassay for aCT quantification in vitro (Figure 2A). Intestinal samples from aCT-treated or not mice were homogenized in PBS and 10 fold diluted in YPD, media that was used to grow the TUP1REP-RFPREP strain for 16 hours at $37^{\circ} \mathrm{C}$. We observed that filaments were present only in samples from aCT-treated mice, while this morphology was absent in standard-antibiotic-treated mice (nonaCT-treated), suggesting that the addition of $1 \mathrm{~g} / \mathrm{L}$ aCT in the drinking water is enough to induce filamentation of the TUP1 REP - RFPREP strain in the mouse intestine.

We next analysed the role of filamentation during colonization. After four days of antibiotic treatment $(2 \mathrm{~g} / \mathrm{L}$ streptomycin, $1 \mathrm{~g} / \mathrm{L}$ bacitracin and $0.1 \mathrm{~g} / \mathrm{L}$ gentamicin) in drinking water, $10^{7} \mathrm{C}$. albicans cells of the TUP1 REP-RFPREP strain were inoculated in a single dose by gavage and fecal samples were analysed. As we can observed in Figure 2B, in the first 7 days TUP1 REP-RFPREP strain colonized similarly to the wt CAF2 strain ( $\left.\approx 10^{7} \mathrm{CFUs} / \mathrm{g}\right)$, as previously described [13]. After this period, the TUP1 ${ }^{\text {REP-RFPREP }}$ colonization levels dropped to $\approx 10^{5} \mathrm{CFUs} / \mathrm{g}$ and were maintained until the end of the experiment ( $\approx 20$ days). At day 20 , aCT was administered in drinking water which led to a significant drop in colonization levels $\left(\approx 10^{3} \mathrm{CFU} / \mathrm{g}\right.$, Figure 2B). Colonization was lost in 5-10 days after the administration of aCT. No CFUs were detected in postmortem analysis of mice, indicating the complete disappearance of the cells in the luminal cavity. These results suggest that the filamentous form is less efficient in gut colonization. When aCT was administered from the beginning of the colonization experiment and before $C$. albicans inoculation, the TUP1 REP-RFPREP strain showed a clear decrease and the colonization ratios began to decrease from day 1 until its complete 
disappearance (Figure 2C), and no candida cells were detected in post-mortem analysis of the mice. Collectively, we conclude that the blockage in a filamentous growth due to TUP1 repression impairs C. albicans to colonize and maintains in the mice gut.

\section{Sensitivity to bile salts and adhesion to the intestine}

Different reasons have been proposed to be the cause for the impairment of gut colonization, such as susceptibility to bile salts or adhesion to the gut mucosa [13]. Sensitivity to bile salts, present in the small intestine, has been proposed as a potential cause to the defects in fitness of candida strains within the gut. For this reason, we performed a drop susceptibility assay with exponentially growing cells of the TUP1REP. RFPREP growing in the presence or absence of aCT to allow yeast and filament forms respectively. However, no significant differences were found compared to the wild type CAF2 strain nor to the filamentation status due to the presence or absent of aCT (Figure 3A).

We also tested the adhesion to the gut mucosa in a competitive ex vivo assay, as previously described [13]. Similar proportions of TUP1 REP-RFPREP, growing in the absence or presence of aCT, and a CAF2-GFP wt strain growing in the same conditions (used as internal control) were mixed and incubated with intestinal mucosa. After 150 minutes, fungal cells were recovered and the relative proportions of both cells type relative to the inoculum was determined as described in Material and Methods. We observed, that the induction of filamentation $(+\mathrm{aCT}$ ) reduced adhesion from $1.25 \pm 0.12$ (mean) to $1.05 \pm 0.18$, although these differences were not statistically significant (Figure 3B).

These results suggest that neither sensibility to bile salt nor adhesion to the gut epithelium explain the defects in colonization associated to TUP1-mediated filamentation.

\section{Discussion}

The controversy on the role of morphology in the virulence of $C$. albicans sustained in the past an intense debate in the mycology field [26-28]. While mutants unable to filament were frequently avirulent in certain animal models [15], it soon became evident that certain hyperfilamentous mutants or strains locked in the filamentous form were also avirulent [16, 21]. The development of genetics soon enabled the construction of strains where hyphal formation could be either triggered or repressed in vivo. These tools demonstrated that hyphal conversion is important for virulence but, more importantly, that ability to alternate between different morphologies was indeed essential for the fungus to cause disease [18]; differences were appreciated between both morphologies and yeast forms were found to colonize more abundantly target organs in the standard mouse systemic model of infection [29]. As the gastrointestinal location may be an important source of endogenous infections [30,31], understanding the mechanisms underlying colonization is essential for the design of efficient prophylactic and therapeutic strategies [11]. In fact, those factors impeding colonization may be relevant strategies to prevent candidiasis.

We have analysed the role of filamentation in commensalism using a TUP1-regulated strain where the addition of doxycycline efficiently leads to filamentation. We first validated the use of this drug in in vivo studies, as doxycycline, a broad spectrum antibacterial used in C. albicans genetics [32] could significantly alter the microbiota of colonized mice. Our data indicate that heat inactivated chlortetracycline (aCT) is as efficient as doxycycline in repressing TUP1 expression. We demonstrate that the TUP1 ${ }^{\text {EEP-RFPREP }}$ strain was indeed able to colonize the mouse gut: initial fungal loads in the gut were $\approx 10^{7} \mathrm{CFU} / \mathrm{g}$ but dropped to $\approx 10^{5}$ after one week and remained constant onwards. 
In this model, induction of filamentation due to TUP1 repression led to a complete loss of $C$. albicans cells in the gut. This result is in agreement with recent data that indicate that filamentous locked strains $(n r g 1 \Delta)$ colonized less efficiently the mouse [19]. However, one important feature of our results is that we introduce cells in the mouse in the yeast form and trigger filamentation inside the host, thus discarding the possibility (not addressed in the mentioned work) that that filamentous forms would be able to colonize once in the intestine but unable to initiate it. This may be relevant as different adaptation phases can be detected when colonizing the mouse gut in this particular commensal model [20]. We still have no explanation for this behaviour; susceptibility to bile salts influences initial colonization loads achieved [33] but no major changes were detected between wt and TUP1REP-RFPREP cells. Transcriptomic analysis of tup1 cells reveals changes in several metabolic traits that could be relevant during adaptation to the gut environment, mainly anaerobic [34] but they are obtained from in vitro cultured cells. Adhesion to the gut epithelium may be also relevant in the context of colonization, as several adhesins are either present or absent in tup1 mutants. It may be as well that the filamentous form is intrinsically less efficient in colonizing due to a mere mechanical reasons (and more efficient removal natural flow of the aliments in the lumen). Our results are in agreement with recent results obtained using germ free mice [35]. These authors identified different $C$. albicans genes (ZFY2, ZCF8, ZFU2 and TRY4) whose deletion resulted in enhanced filamentation and reduced colonization. In addition, expression of the UME6 filamentation regulator had a similar effect. As these experiments were obtained with germ free mice, mice microbiota does not seem to influence the preference of yeast versus filaments in the mouse lumen. The reasons for this may be speculative at this stage but a different immune response, resistance to innate antifungal mechanisms, adherence to the lumen or even simple mechanical features may be responsible for this effect. Therefore, our work supports that the $C$. albicans yeast form is more prone to colonize than hyphae. Importantly, our results reinforce the concept that induction of filamentation of $C$. albicans in the human gut via drugs or food products may be an alternative approach to reduce colonization and minimize systemic fungal infections caused by this yeast.

\section{Conclusions}

The work presented here show that induction of filamentation via TUP1 repression results in a loss of C. albicans cells within the mouse gut, while the corresponding yeast forms efficiently colonizes at lower levels. These effects are not due to a defect in adhesion to the gut epithelium nor to an increased susceptibility to bile salt suggesting that filamentous forms are intrinsically less adapted to grow in the mammalian intestines.

\section{Summary Points}

TUP1 repression led to a filament locked phenotype both in liquid and solid media

Autoclaved chlortetracycline, aCT, has a reduced antimicrobial effect but is still able to regulate the TETON/TETOFF regulatable system in Candida albicans

TUP1 repression renders candida filament cells that are unable to establish in the murine gut

The induction of filamentation by repressing TUP1 induced the loss of candida cells once stablished in the murine gut 
No differences in susceptibility to bile salt nor to the ability to adhere to the gut mucosa is due to the repression of TUP1

\section{References}

1. Navarro-García F, Sánchez M, Nombela C, Pla J. Virulence genes in the pathogenic yeast Candida albicans. FEMS Microbiological Reviews 25(2), 245-268 (2001).

2. Berman J, Sudbery PE. Candida albicans: a molecular revolution built on lessons from budding yeast. Nature Reviews in Genetics 3(12), 918-930 (2002).

3. Perez-Nadales E, Nogueira MF, Baldin $\mathrm{C}$ et al. Fungal model systems and the elucidation of pathogenicity determinants. Fungal Genet. Biol. 70, 42-67 (2014).

4. Naglik JR, Fidel PL, Jr., Odds FC. Animal models of mucosal Candida infection. FEMS Microbiological Letters 283(2), 129-139 (2008).

5. Kinneberg KM, Bendel CM, Jechorek RP et al. Effect of INT1 gene on Candida albicans murine intestinal colonization. Journal of Surgery Research 87(2), 245-251 (1999).

6. Bendel CM, Wiesner SM, Garni RM, Cebelinski E, Wells CL. Cecal colonization and systemic spread of Candida albicans in mice treated with antibiotics and dexamethasone. Pediatr. Res. 51(3), 290-295 (2002).

7. Koh AY, Kohler JR, Coggshall KT, Van RN, Pier GB. Mucosal damage and neutropenia are required for Candida albicans dissemination. PLoS Pathog. 4(2), e35 (2008).

8. Koh AY. Murine models of Candida gastrointestinal colonization and dissemination. Eukaryotic Cell 12(11), 1416-1422 (2013).

9. Prieto D, Correia I, Pla J, Roman E. Adaptation of Candida albicans to commensalism in the gut. Future Microbiol. 11, 567-583 (2016).

10. Neville BA, D'enfert C, Bougnoux ME. Candida albicans commensalism in the gastrointestinal tract. FEMS Yeast Res 15(7), (2015).

11. Noble SM, Gianetti BA, Witchley JN. Candida albicans cell-type switching and functional plasticity in the mammalian host. Nat Rev Microbiol, (2016).

12. Noble SM. Candida albicans specializations for iron homeostasis: from commensalism to virulence. Curr. Opin. Microbiol. 16(6), 708-715 (2013).

13. Prieto AD, Román E, Correia I, Pla J. The HOG pathway is critical for the colonization of the mouse gastrointestinal tract by Candida albicans. PLoS One 9(1), e87128 (2014).

14. Perez JC, Kumamoto CA, Johnson AD. Candida albicans commensalism and pathogenicity are intertwined traits directed by a tightly knit transcriptional regulatory circuit. PLOS Biol. 11(3), e1001510 (2013).

15. Lo HJ, Kohler JR, Didomenico B, Loebenberg D, Cacciapuoti A, Fink GR. Nonfilamentous Candida albicans mutants are avirulent. Cell 90(5), 939-949 (1997).

16. Alonso-Monge $\mathrm{R}$, Navarro-García $\mathrm{F}$, Molero $\mathrm{G}$ et al. Role of the mitogen-activated protein kinase Hog1p in morphogenesis and virulence of Candida albicans. J. Bacteriol. 181(10), 3058-3068 (1999).

17. Saville SP, Lazzell AL, Monteagudo C, Lopez-Ribot JL. Engineered control of cell morphology in vivo reveals distinct roles for yeast and filamentous forms of Candida albicans during infection. Eukaryotic Cell 2(5), 1053-1060 (2003).

18. Saville SP, Lazzell AL, Chaturvedi AK, Monteagudo C, Lopez-Ribot JL. Use of a genetically engineered strain to evaluate the pathogenic potential of yeast cell and filamentous forms during Candida albicans systemic infection in immunodeficient mice. Infect. Immun. 76(1), 97-102 (2008).

19. Vautier S, Drummond RA, Chen $\mathrm{K}$ et al. Candida albicans colonization and dissemination from the murine gastrointestinal tract: the influence of morphology and Th17 immunity. Cell. Microbiol. 17(4), 445-450 (2015).

20. Prieto $\mathrm{D}, \mathrm{Pla}$ J. Distinct stages during colonization of the mouse gastrointestinal tract by Candida albicans. Front Microbiol 6, 792 (2015). 
21. Braun BR, Johnson AD. Control of filament formation in Candida albicans by the transcriptional repressor TUP1 Science 277(5322), 105-109 (1997).

22. Correia I, Roman E, Prieto D, Eisman B, Pla J. Complementary roles of the Cek1 and Cek2 MAP kinases in Candida albicans cell-wall biogenesis. Future Microbiol. 11, 51-67 (2016).

23. Urrialde V, Prieto D, Pla J, Alonso-Monge R. The Pho4 transcription factor mediates the response to arsenate and arsenite in Candida albicans. Front Microbiol 6, 118 (2015).

24. Román E, Nombela C, Pla J. The Sho1 adaptor protein links oxidative stress to morphogenesis and cell wall biosynthesis in the fungal pathogen Candida albicans. Mol. Cell. Biol. 25(23), 1061110627 (2005).

25. Bochner BR, Huang HC, Schieven GL, Ames BN. Positive selection for loss of tetracycline resistance. J. Bacteriol. 143(2), 926-933 (1980).

26. Kobayashi GS, Cutler JE. Candida albicans hyphal formation and virulence: is there a clearly defined role? Trends Microbiol. 6(3), 92-94 (1998).

27. Mitchell AP. Dimorphism and virulence in Candida albicans. Curr. Opin. Microbiol. 1(6), 687-692 (1998).

28. Liu H. Co-regulation of pathogenesis with dimorphism and phenotypic switching in Candida albicans, a commensal and a pathogen. Int. J. Med. Microbiol. 292(5-6), 299-311 (2002).

29. Saville SP, Lazzell AL, Bryant AP et al. Inhibition of filamentation can be used to treat disseminated candidiasis. Antimicrob. Agents Chemother. 50(10), 3312-3316 (2006).

30. Odds FC, Davidson AD, Jacobsen MD et al. Candida albicans strain maintenance, replacement, and microvariation demonstrated by multilocus sequence typing. J. Clin. Microbiol. 44(10), 36473658 (2006).

31. Miranda LN, Van Der Heijden IM, Costa SF et al. Candida colonisation as a source for candidaemia. J. Hosp. Infect. 72(1), 9-16 (2009).

32. Park YN, Morschhauser J. Tetracycline-inducible gene expression and gene deletion in Candida albicans. Eukaryotic Cell 4(8), 1328-1342 (2005).

33. Prieto D, Roman E, Alonso-Monge R, Pla J. Overexpression of the Transcriptional Regulator WOR1 Increases Susceptibility to Bile Salts and Adhesion to the Mouse Gut Mucosa in Candida albicans. Front Cell Infect Microbiol 7, 389 (2017).

34. Kadosh $\mathrm{D}$, Johnson $\mathrm{AD}$. Induction of the Candida albicans filamentous growth program by relief of transcriptional repression: a genome-wide analysis. Mol. Biol. Cell 16(6), 2903-2912 (2005).

35. Krysan DJ, Böhm L, Torsin $S$ et al. The yeast form of the fungus Candida albicans promotes persistence in the gut of gnotobiotic mice. PLoS Pathog. 13(10), (2017).

\section{Financial disclosure/Acknowledgements}

This research is supported by grants BIO2015-6477. The authors have no other relevant affiliations or financial involvement with any organization or entity with a financial interest in or financial conflict with the subject matter or materials discussed in the manuscript apart from those disclosed.

\section{Ethical conduct of research}

The authors state that they have obtained appropriate institutional review board approval or have followed the principles outlined in the Declaration of Helsinki for all human or animal experimental investigations. All experiments involving animals performed in this work were carried out in strict accordance with the regulations in the "Real Decreto 1201/2005, BOE 252" for the Care and Use of Laboratory Animals of the "Ministerio de la Presidencia," Spain. The protocol used in the commensalism model was approved by the Animal Experimentation Committee of the University Complutense of Madrid (CEA 33-2015) and Comunidad de Madrid according to Artículo 34 del RD 53/2013 (PROEX 226/15). The treatments did not result in noticeable disease in the animals; nevertheless, all procedures were conducted minimizing any suffering. The number of animals per experiment was adjusted to a minimum for ethical reasons. 

Figure 1. TUP1-myc regulation in regulated strains under the control of the TET-OFF system.

A) Cellular morphology after 16 hours at $37^{\circ} \mathrm{C}$ of growth in the absence (-) or presence of $20 \mathrm{mg} / \mathrm{L}$ doxycycline. An image from a drop assay on solid media with or without dox after $48 \mathrm{~h}$ growth is shown (inset); B) Cellular morphology of the TUP1REP-RFPREP strain in YPD supplemented with increasing concentration of dox after 16 hours of growth at $37^{\circ} \mathrm{C}$. C) Tup1-myc detection under different doxycycline (upper panel) or aCT (lower panel) concentrations in YEPD at $37^{\circ} \mathrm{C}$

\section{Figure 2. Filamentous forms are less efficient to colonize de GI tract}

A) Bioassay to detect the aCT presence in mice intestine. Cellular morphology of the TUP1 ${ }^{\text {REP }}$-RFPREP strain in the presence of the intestine content from mice treated or not with aCT after 16 hours of growth at $37^{\circ} \mathrm{C}$., B) TUP1 REP-RFPREP colonization rates expressed as logarithmic values of the CFUs per gram of stool along the time in mice treated with standard antibiotic treatment. At day $20 \mathrm{aCT} 1 \mathrm{~g} / \mathrm{L}$ was added to the drinking water; C) TUP1 REP-RFPREP colonization rates in mice treated with standard antibiotic cocktail plus aCT $1 \mathrm{~g} / \mathrm{L}$ from the beginning of the experiment.

\section{Figure 3. Analysis of bile salt sensitivity and adhesion to mouse mucosa}

A) Tenfold serial dilutions of overnight growing cultures from the indicated strains were spotted in YPD plates supplemented or not with bile salts in the presence or not of aCT $20 \mathrm{mg} / \mathrm{L} ; \mathrm{B}$ ) The adhesion relative index (ARI) was determined for the TUP1REP-RFPREP in the large intestine in the presence or absence of aCT. Individual values are shown with the mean \pm SEM. 
A)

CAF2

TUP1REP-RFPREP

tup1
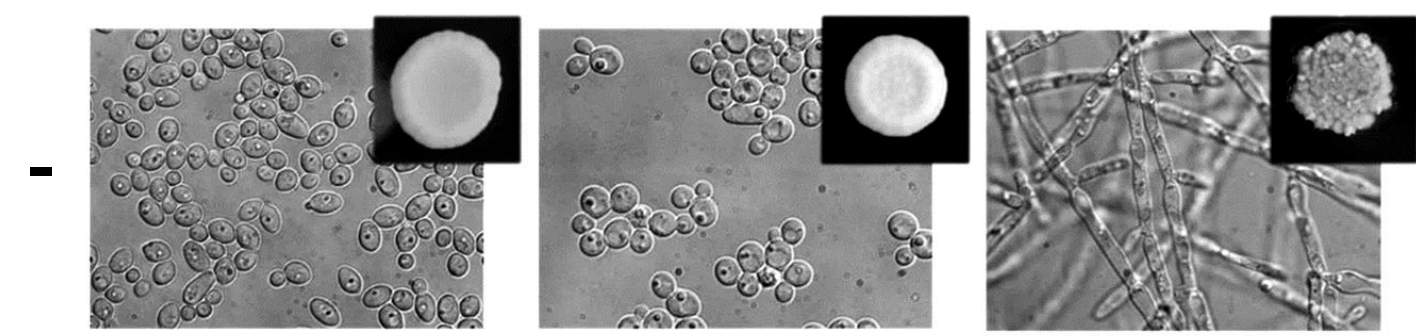

Dox

(20 mg/L)
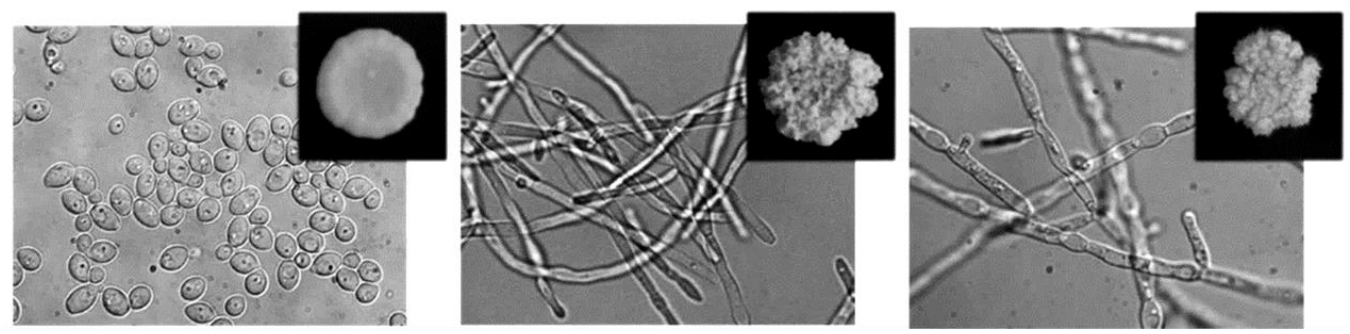

B)

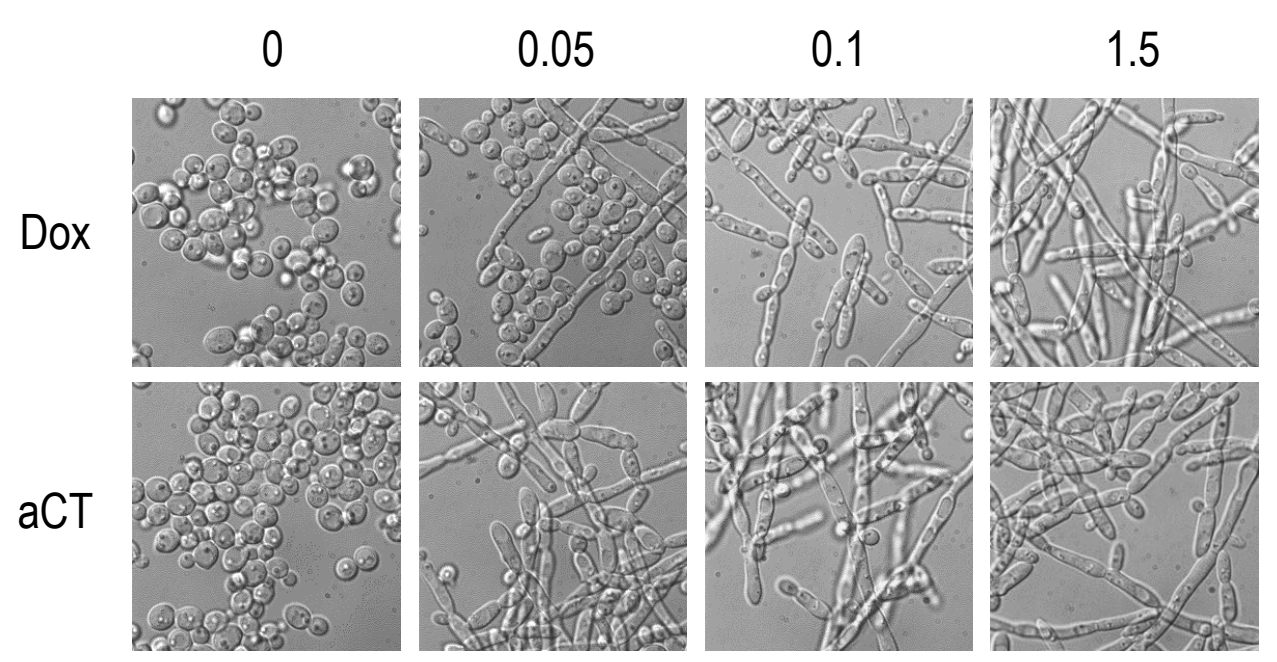

C)

Tup1-myc

(59 kDa)
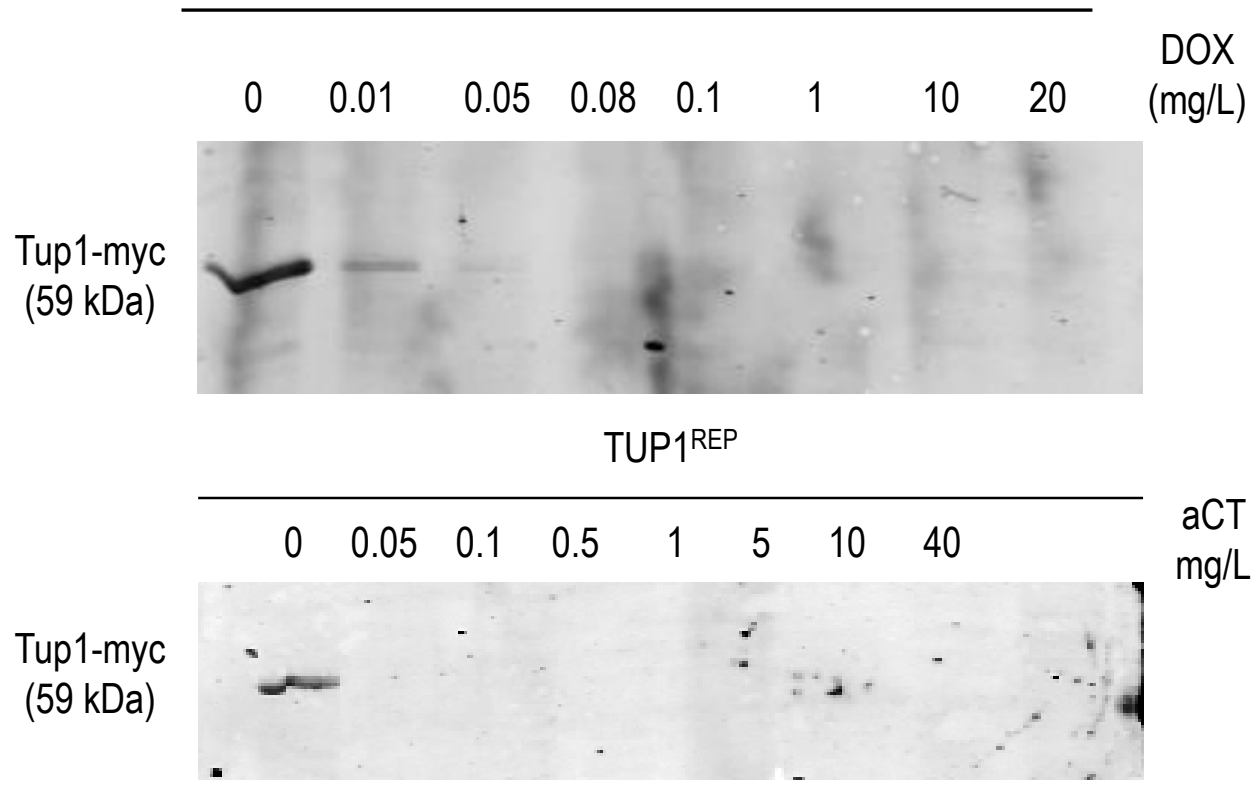
A)
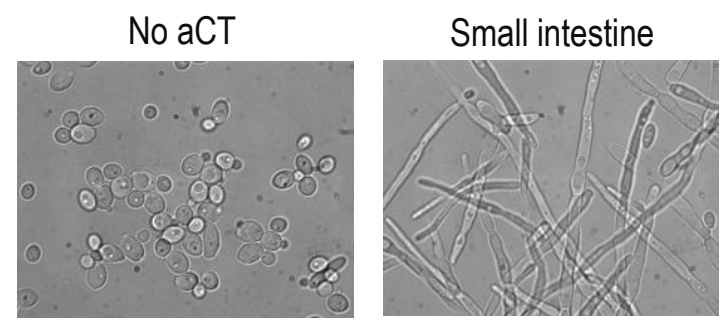
Large intestine

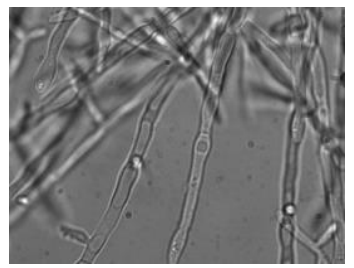

B)

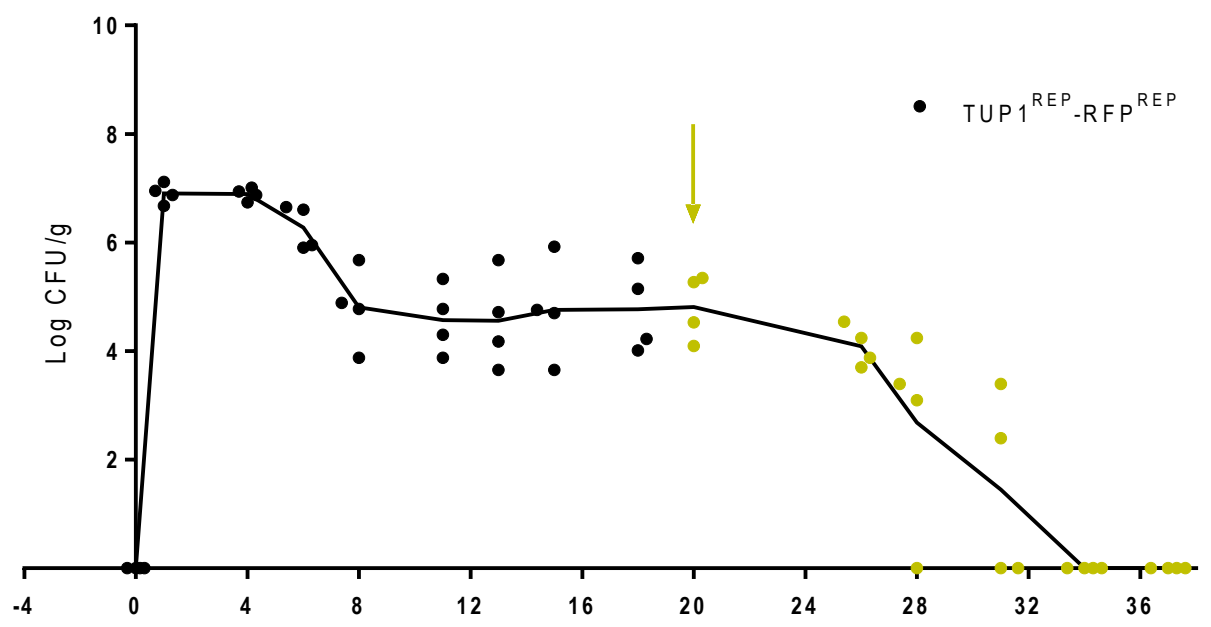

Time (days)

C)

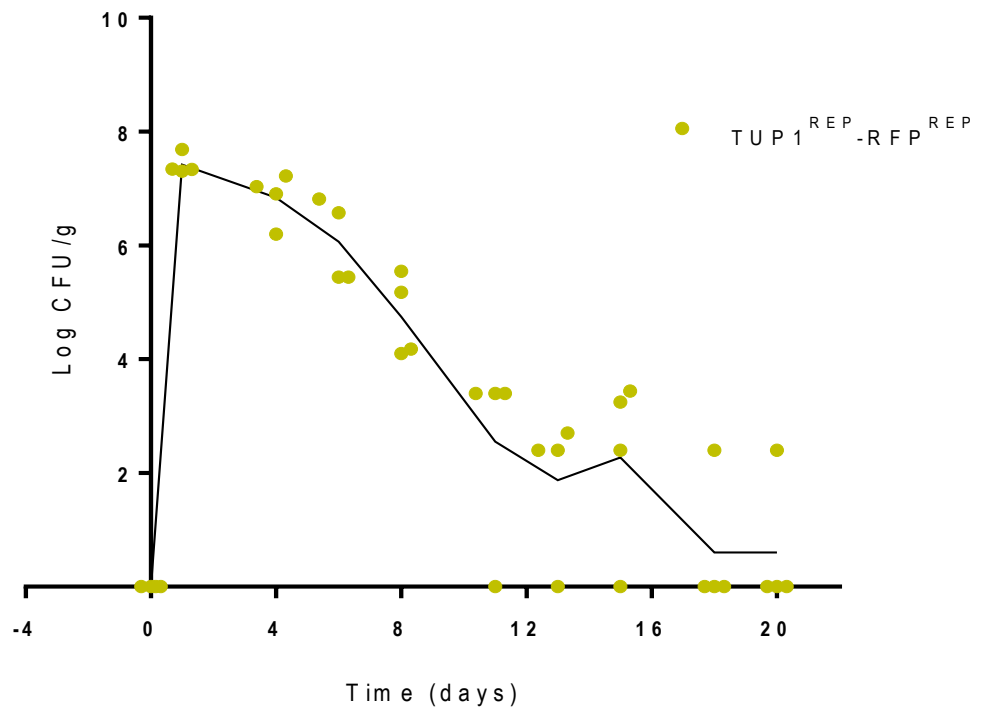


A)

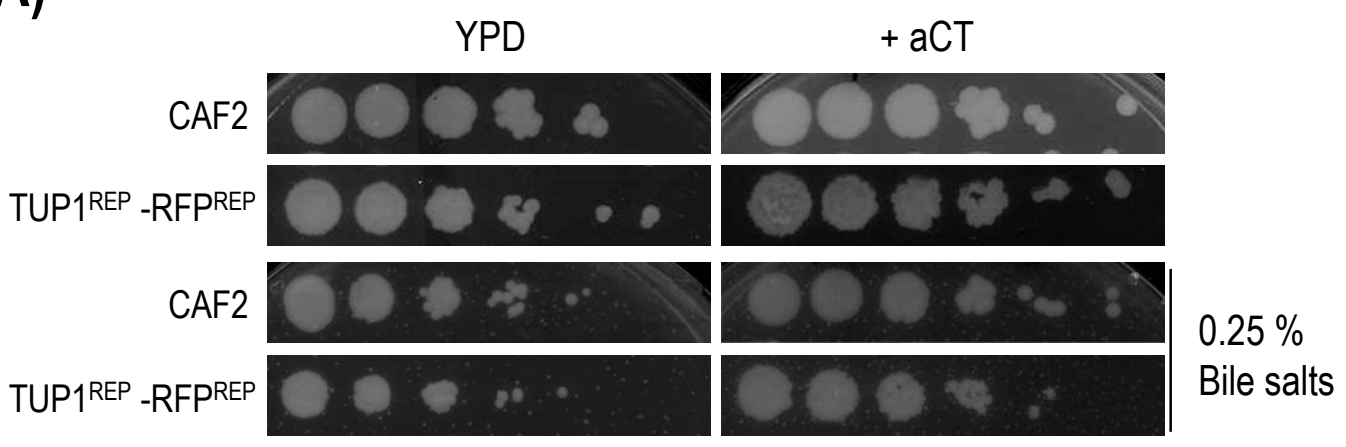

B)

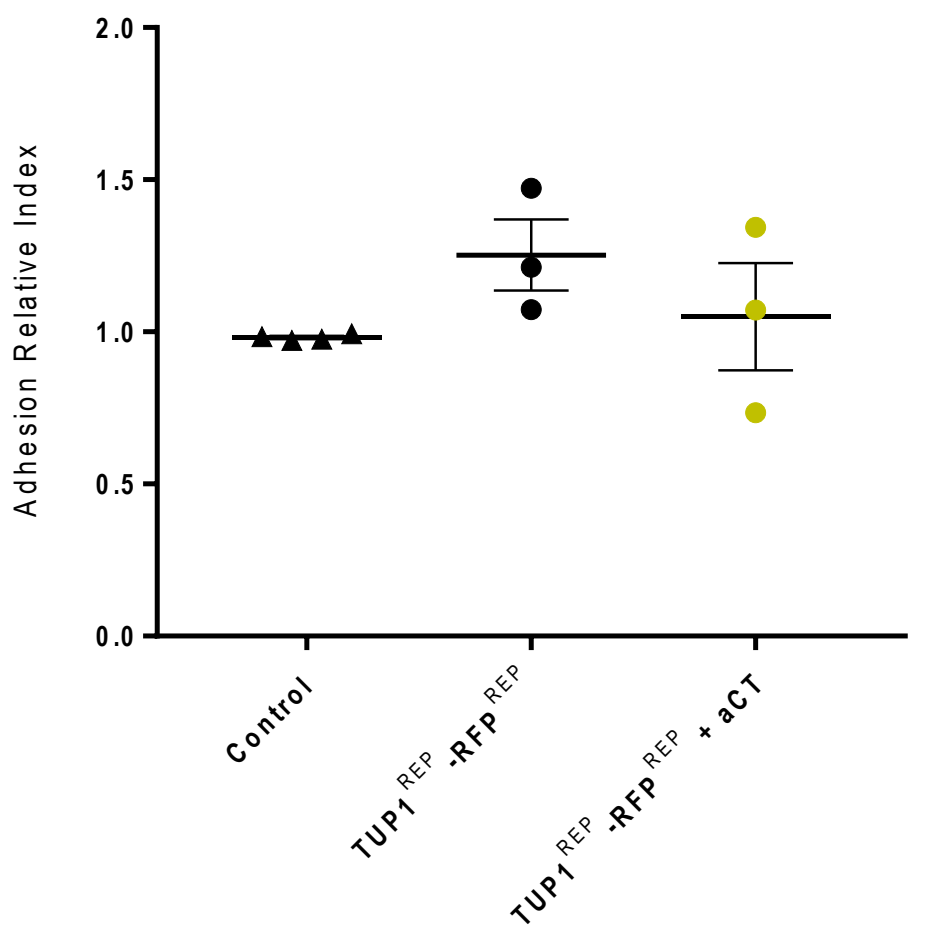

